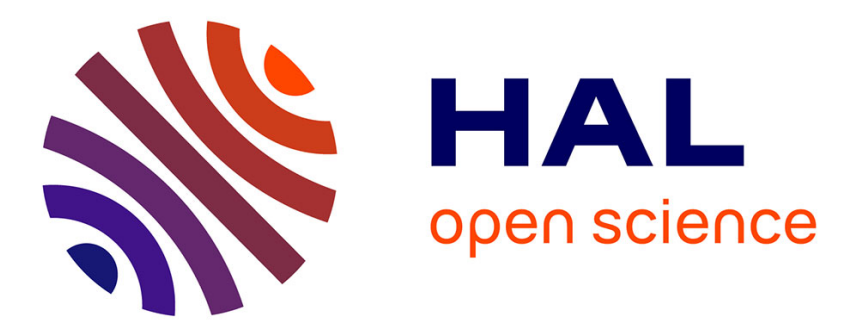

\title{
Interoperability as a Key Concept for the Control and Evolution of the System of Systems (SoS)
}

\author{
Stéphane Billaud, Nicolas Daclin, Vincent Chapurlat
}

\section{To cite this version:}

Stéphane Billaud, Nicolas Daclin, Vincent Chapurlat. Interoperability as a Key Concept for the Control and Evolution of the System of Systems (SoS). 6th International IFIP Working Conference on Enterprise Interoperability (IWEI), May 2015, Nîmes, France. pp.53-63, 10.1007/978-3-662-471579_5. hal-01438407

\author{
HAL Id: hal-01438407 \\ https://hal.inria.fr/hal-01438407
}

Submitted on 17 Jan 2017

HAL is a multi-disciplinary open access archive for the deposit and dissemination of scientific research documents, whether they are published or not. The documents may come from teaching and research institutions in France or abroad, or from public or private research centers.
L'archive ouverte pluridisciplinaire HAL, est destinée au dépôt et à la diffusion de documents scientifiques de niveau recherche, publiés ou non, émanant des établissements d'enseignement et de recherche français ou étrangers, des laboratoires publics ou privés. 


\title{
Interoperability as a key concept for the control and evolution of the System of Systems (SoS)
}

\author{
Stéphane Billaud ${ }^{1}$, Nicolas Daclin ${ }^{1}$, Vincent Chapurlat ${ }^{1}$ \\ ${ }^{1}$ LGI2P, , Ecole des Mines d'Alès, Parc Scientifique G. Besse, 30035 Nîmes Cedex 1, \\ France \\ surname.name@mines-ales.fr
}

\begin{abstract}
A coalition of enterprises wanting to collaborate, and more generally a Collaborative Network of Organizations (CNO), can conceptually be assimilated as a System of Systems (SoS) presenting a number of characteristics to respect all over its life cycle. Interoperability is one of these characteristics (both functional and non-functional), which is from our point of view, essential in order to guarantee the control of the SoS, its behavior and the fulfillment of its mission(s). Moreover, it ensures the reaction of the SoS to deal with some risky situations and with potential local or global deficits during its functioning. In this paper, we propose to determine the relation between the current level of interoperability of the SoS and its functioning whatever may be its situation. A matrix shows how this relation evolves taking into account several characteristics of the SoS, particularly its capacity to respect interoperability requirements (Compatibility, Interoperation, Autonomy and Reversibility) and the so-called analysis perspectives of the SoS: Performance, Integrity and Stability. This relation is requested in order to permit and to guide SoS behavioral simulation currently in development. Thus, a set of indicators is derived and formalized.
\end{abstract}

Keywords: Interoperability, System of Systems (SoS), System of Systems Engineering (SoSE), Performance, Stability, Integrity, Adaptability.

\section{Introduction}

The following definition seems to adequately encapsulate the multiple definitions that have been given to the concept of System of Systems and will be useful for the remainder of this paper. A System of Systems (SoS) is a set of heterogeneous and existing subsystems assembled together to achieve a global mission that a system alone cannot fulfil, while maintaining the operational and managerial independency (autonomy) of each of the subsystems. These subsystems have then to be able to communicate and to work harmoniously together or to adapt their behavior and functioning locally when facing any evolution of the environment of the SoS [1], [2], [3], [4], [5]. It is admitted that the SoS Engineering (SoSE) activities carefully focus on choosing and assembling these subsystems as well as designing appropriate interfaces to facilitate this assembling [6]. Subsystems are selected and involved according to their potential roles, available resources, competences and 
know-how that can be shared in order to fulfil the SoS mission. Particular attention is given to some constraints that have to be also considered especially the capacity of subsystems of being interoperable. Indeed, for instance DoDAF [7] and System Engineering [8], [9] claim that interoperability is required to coordinate and make efficient such large multi-disciplinary and heterogeneous coalition of subsystems. Therefore, interoperability has to be fully considered when the chosen subsystems are assembled for a more or less short period during which they will have to work together, share flows, data and resources in order to build their SoS.

Moreover, a strong linkage exists between the interoperability and the so-called analysis perspectives namely Stability, Integrity and Performance [6]. Therefore, and to address this challenge, the original aim of this paper is to investigate the impact of the interoperability on the so-called analysis perspectives by proposing an impact matrix and by defining a set of indicators that characterizes and helps to understand this impact. With respect to the state-of-the-art and to the best of our knowledge, the requested interoperability of heterogeneous subsystems has not been yet handled before and this paper characterizes the novelty of the approach. It is evidently a new challenging area and there are research directions towards discovering it [10].

This paper focuses first on the reasons behind considering the interoperability as a crucial characteristic of the SoS in order to control it and to help it achieving its mission throughout its evolution and in various situations that might take risky aspects. Afterwards, we define a set of requirements that allow us characterizing the requested subsystems' interoperability. Moreover, relationship between subsystems' interoperability and SoS' analysis perspectives is presented. The first result is a matrix aiming to assist the engineers, designers and managers involved in SoSE process in choosing their subsystems prior the assembling and understanding the impact of the interoperability over the SoS analysis perspectives all over its life cycle. This will be done by evaluating this impact through a simulation technique not described here. Therefore, a set of indicators is defined in order to concretize the existing relationship between the interoperability and the SoS analysis perspectives. These indicators are not exhaustive but in our point of view, the selected ones are complete and enough to allow the evaluation of the impact of interoperability on the SoS analysis perspectives.

\section{Interoperability}

\subsection{Interoperability vs. SoS characteristics}

Interoperability is defined recurrently in the literature in a way to provide a better understanding of its various aspects and levels [11]. Thus, our attention is directed to define interoperability as the ability of connected, autonomous, "loosely coupled" and possibly heterogeneous systems to coexist, to interoperate and to exchange flows (data and services, material or energy) to/from other systems while continuing their own logic of operation preserving their autonomy. 
In essence, this definition reveals various characteristics, which are consistent with the SoS expected characteristics. The autonomy of a subsystem i.e. the possibility to continue to act and make decisions, in order to ensure its own mission independently of other subsystems, is consistent with the expected managerial independency of the subsystems. The reversibility of a relation between two subsystems that allows a subsystem to achieve its mission, after breaking an alliance with other subsystems composing the same SoS, is coherent with the requested operational independency of the subsystems. The subsystems are seen as "loosely coupled". On the one hand, this kind of coupling enhances the connectivity which characterizes a SoS, where subsystems are capable of building links among their interfaces and destroying them dynamically [12] and on the other hand, it enhances the evolutionary development of the SoS [3] when it becomes possible to easily remove, modify or add subsystems from the SoS. The heterogeneity of the subsystems is essential for the SoS since it can only achieve its global mission by leveraging the diversity of its subsystems [12].

Thus, maintaining a sufficient level of interoperability of each subsystem helps the preservation of these SoS characteristics (constituent subsystems autonomy, enriched connectivity and commitment to diversity of subsystems) and the SoS behavior.

Last, SoS passes through various stages in its life cycle (See Figure 1) during which these characteristics may evolve but have to be maintained in an acceptable range.

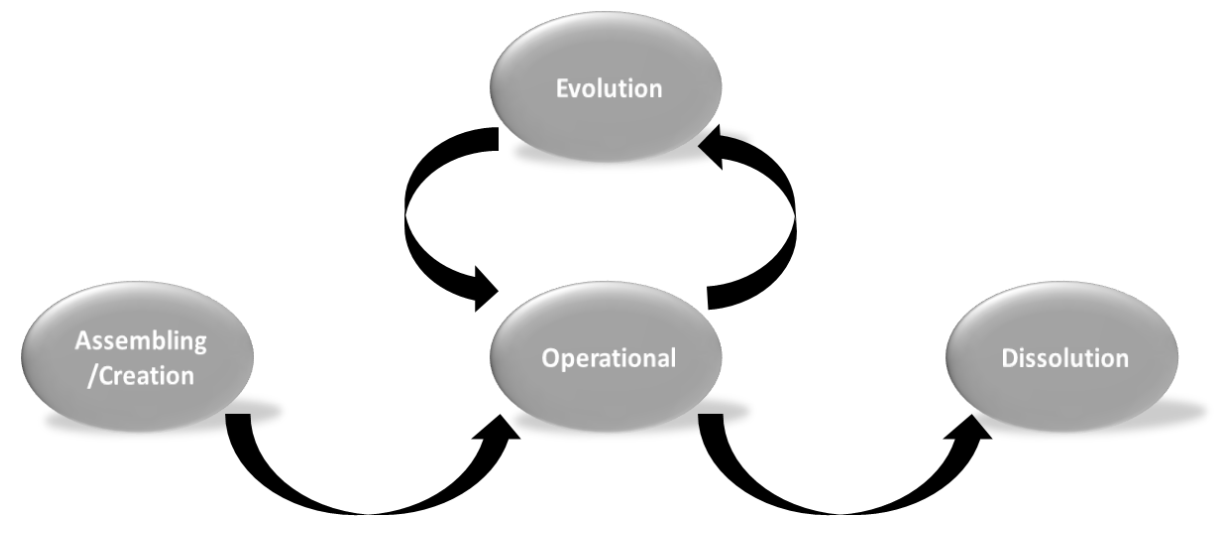

Figure 1 Life cycle of a SoS [6]

\subsection{Interoperability vs. SoS analysis perspectives}

Beyond the classical System Engineering approach, System of Systems Engineering (SoSE) puts emphasis on the selection of relevant subsystems taking into consideration the necessity of staying interoperable in order to participate efficiently in the global mission of the SoS.

In this way, it is required to formalize the design of a SoS by taking into consideration the relationships between subsystems' interoperability and some functional characteristics as non-functional of a SoS. We focus here on the impact of subsystems interoperability on non-functional characteristics namely analysis perspectives (Stability, Integrity and Performance) as demonstrated hereafter. 
Stability reflects the ability of a SoS to maintain its viability and to adapt (e.g. its structure or its behavior i.e. this requests generally adaptation of concerned subsystems) to any change in its environment [6], [13]. It characterizes the cohesive relationship that should exist between the system structure and its activities or programs which define what the system should do. The stability level of the SoS must evolve when subsystems have to deal with some risky and/or unexpected situations whether they are due to internal or external events. Stability has six basic concepts which are generic for any SoS: Constancy, Resilience, Persistence, Resistance, Elasticity, and Domain of attraction [14]. Currently, none of the existing approaches (known as architectural styles of self-adaptation) enables a SoS to reason about itself and adapt to achieve particular stability, performance or quality objectives in the face of uncertainties and changes. The only existing architectural style of self-adaptation handles the stability from a local point of view of the subsystems without taking into consideration the subsystems interoperability that can impact positively or negatively the stability [15].

Integrity reflects the ability of a SoS to return to a known functioning and operating mode in case of any local change in its existing configuration (e.g. loss of one or more resources, or non-expected or even emergent situation due to subsystems interactions). When one or more subsystems of the SoS leaves the SoS, these subsystems have to continue to maintain their own operations and the remaining group of subsystems should continue to operate in the new context of the SoS.

Performance reflects the SoS ability to achieve its mission by reaching its objectives in terms e.g. of costs, duration, quality of service etc. It characterizes the relationship between the functions that have to be executed by the system and the compliance of the services provided by the resources for example, through indicators of time, quality and costs that reflect the efficiency, effectiveness and the relevance of the involved set of resources [16]. The goal is not to guarantee a maximum level of performance, but to be able to return to a predefined level of performance after an external or internal change (addition, deletion, modification of a subsystem or an interaction etc.).

Evaluating the impact of interoperability on the analysis perspectives requests first to identify interoperability requirements and constraints allowing us to overcome the three classical barriers of interoperability detailed in [17]: Conceptual, Technological and Organizational.

Improving both conceptual and technical interoperability is important to support organizational interoperability. These barriers take place in four areas of concerns of the SoS: flows (carrying out data, material or energy), services, processes and business. It is important to consider the three barriers to draw the interoperability requirements in order to allow proactive anomaly detection at the three levels (conceptual, technical and organizational).

The first basic interoperability requirement concerns the subsystems compatibility. The compatibility refers here to the interfaces imposed by the interactions between the subsystems. Interfaces can be technical, organizational, HMI or logical at high level of abstraction. Imposing standards interfaces or well-defined interfaces and common integration mechanisms are not always the solution in dynamic environment that can be considered as a SoS [18], [19]. 
The second basic interoperability requirement concerns the subsystems' autonomy. A subsystem must effectively respects the expected objectives, stakeholders' requirements and constraints defined for the SoS (e.g. cost, delay, quality) but meanwhile, it should respect its own requirements.

During the operational phase of the SoS, subsystems' interoperation appears as another interoperability requirement. It concerns, the ability of a collection of subsystems to share or exchange specified information/energy/material in order to achieve a specified purpose/mission in a given context.

In the dissolution phase of the SoS, the inter subsystems' relations (reversibility) is an important interoperability requirement. For instance, once a set of subsystems break the alliance with each other, each subsystem must be able to return to a state in which it reaches at least its original level of performance while executing its usual operations and consequently it respects its own requirements.

Last, interoperability requirements vary from one SoS type to another. For instance Directed SoS is considered here whereas the SoS requires to have an authority and a management role over its group of subsystems while preserving their ability to operate independently.

\subsection{Interoperability measurement}

Interoperability has been studied in multiple fields [20], [21] and various approaches have been proposed to measure and evaluate the interoperability level of a system whatever may be its nature and sometimes its complexity. These approaches are mainly based on maturity measurement. A recent survey presented fourteen interoperability models used to measure the interoperability [11]: Spectrum of Interoperability Model (SoIM) [22], Quantification of Interoperability Methodology (QoIM) [23], Military Communications and Information Systems Interoperability (MCISI) [24], Levels of Information System Interoperability Model (LISI) [25] (this model is similar to SoIM, it is suited and adapted for measuring information systems interoperability), Interoperability Assessment Methodology (IAM) [26] (this model is similar to QoIM), Organizational Interoperability Maturity Model for C2 (OIM) [27](This model is an extension of the LISI model), Stoplight [28], Levels of Conceptual Interoperability Model (LCIM) [29](this model is similar to LISI and OIM, however it is used in the conceptual design to prove if meaningful interoperability between the systems is possible), Layers of Coalition Interoperability (LCI) [30], NATO C3 Technical Architecture Reference Model for Interoperability (NMI)[31], System-of-Systems Interoperability Model (SoSI) [32] (SoSI was proposed to support the Software Engineeringùùu* Institutes SoS interoperability research. However, it does not contain specific metrics to quantify interoperability within a SoS), Non-Technical Interoperability Framework (NTI)[33] (this model is based on the OIM organizational model), Organizational Interoperability Agility Model (OIAM) [34] (it builds upon the OIM organizational model), The Layered Interoperability Score (i-Score)[35] (This model is a mathematical method made in order to measure the interoperability of all types of systems for a very specific operational scenario/thread). 
After presenting the approaches mentioned previously, we realize that all of them focus on a specific application domain (the interoperability of information systems) and only few approaches integrate the organizational aspects of interoperability. Moreover, none of these interoperability measurement approaches has been presented or tested in large systems or organizations like the SoS and [11] did not present any evidence of that. Therefore, the approach presented in the next section, offers an evaluation matrix that allows measuring the interoperability impacts, in a large and complex context (SoS), on the SoS analysis perspectives.

\section{Interoperability impact matrix}

The impact matrix aims to present a new approach of the interoperability-impact analysis which combines the interoperability sub requirements and their impact on the SoS analysis perspectives. Therefore, it is divided into two main axes: (I) the interoperability (Compatibility, Interoperation, Autonomy and Reversibility) and (II) the SoS analysis perspectives (Performance and Adaptability). The integrity and stability of the SoS are combined into one characteristic that we call: the SoS' adaptability. It is the ability of a SoS to adapt to any new situation or change and to return to a known operating or functioning mode whatever the changes result from internal (integrity) or external (stability) causes. It will be evaluated based on its six concepts: Constancy, Resilience, Persistence, Resistance, Elasticity, and Domain of attraction mentioned in Section 2.2.

Mainly, two subsystems inside a SoS are at a high level of interoperability if and only if they are at a high level of Compatibility, Interoperation, Autonomy and Reversibility.

Compatibility, Compatibility means to harmonize subsystems in order to be ready to collaborate. Compatibility focuses on a static point of view of the collaboration and remains insufficient to determine if the subsystems are interoperable during the SoS life cycle. It is necessary to consider the evolution of the context and of the situation of each subsystem. A set of indicators is required to analyze the impact of the compatibility between the subsystems on the performance of the SoS. They are divided into subgroups according to the kind of compatibility.

1. Organizational and conceptual compatibility indicators: syntax (the information to be exchanged is expressed with the same syntax?), preparedness (the data are well defined and documented?), Understanding (communication and shared information rate), Command style (are authorities/responsibilities are clearly defined?) and trust.

2. Technical compatibility indicators: Common Operating Environments, Standard procedures and training, standard complaint, Basic data format (Information exchange is restricted to homogeneous data exchange), Media format, Applications, security profile (a security profile contains information that governs at what security level(s) a system may operate), Media exchange procedures, System services, data, Heterogeneous information (This form of information represents data repositories that contain more than one data format) and Information space. 
3. Operational and behavioral compatibility indicators: compatibility with prior experience, compatibility with existing work practices and compatibility with preferred work style.

4. Functional compatibility: response and execution time.

In this case, compatibility impacts each perspective as follows:

Compatibility $\rightarrow$ Performance: The compatibility between subsystems impacts the SoS performance. In the absence of compatibility of interfaces for example, it becomes impossible for interactions to take place, therefore the system will be unable to fulfill its mission(s) and by consequence unable to reach its performance's objectives. Furthermore, a low level of compatibility implies a limited interaction which will induce a lower performance.

On the one hand, an increase in the compatibility between the subsystems imposes sometimes constraints to respect. These constraints imply a decrease in the performance of the SoS. On the other hand, an increase in the compatibility might be useless if the subsystems reached already a sufficient level of compatibility to guarantee the predefined level of performance for the SoS. In this case, the performance will not vary with the increase of the compatibility.

A decrease in the compatibility between the subsystems prevents or limits the exchange of data/material/energy etc., this induces a decrease in the performance of the SoS. However, if the subsystems need a level of compatibility less than the actual one in order to perform perfectly, then a decrease of the compatibility will have no impact on the level of the predefined performance since their level of compatibility was higher than requested and they can absorb a decrease in the compatibility on condition that it remains sufficient to keep the necessary level to perform adequately.

Compatibility $\rightarrow$ Adaptability: An increase or decrease in the compatibility following an add, remove or modification of a subsystem or an interaction between the subsystems might induce a decrease in the performance of the SoS since the subsystems take a certain time to reach again an accepted level of compatibility necessary to get to the predefined level of performance. The level of performance might stay stable if the subsystems are able to adapt rapidly.

Interoperation, is the ability of a collection of communicating systems to share or exchange specified information/energy/material in order to achieve a specified purpose/mission in a given context. It is measured through a set of indicators which seem to be only adequate to a specific types of telecommunication SoS. However, these indicators can be applied to any kind of SoS since the flow between subsystems can be data, information, material or energy. Therefore, these indicators are not limited to the domain of telecommunication:

1. Time of interoperation: The time of interoperation corresponds to the duration between the date when information is requested and the date when the requested information is used

2. Quality of interoperation: The quality of interoperation takes in consideration three kinds of quality: (1) the quality of exchange (The quality of exchange draws up if the exchange is correctly performed), (2) the quality of use (The quality of use represents the number of information received by a partner in comparison with the number of information requested.) and, (3) the quality of conformity (The quality of conformity corresponds to the exploitation of the information) 
3. Capacity: is the rate at which data may be passed over time

4. System overload: when more data must be exchanged than the system is able to transmit

5. Underutilization: when the system data rate/message load is less than its full capacity but messages are waiting in queues to be transmitted

6. Under capacity: when messages remain in queues and the system data rate is at the maximum

7. Data latency: is the elapsed time from the transmission to the reception

Then, interoperation impacts each perspective as follows:

Interoperation $\rightarrow$ Performance: The interoperation between the subsystems impacts the SoS performance. In the absence of the interoperation, the subsystems are no longer able to exchange data/material/energy etc. therefore the SoS cannot achieve its mission(s) neither its performance's objectives. Moreover, a subsystem with a very low capacity limits the interoperation with another subsystem with higher capacity; therefore the performance of the overall system (SoS) will be impacted.

An increase or decrease in the interoperation between the subsystems might have three different impacts on the performance of the SoS. It decreases the SoS performance if we exceed the capacity of the subsystems to absorb the high rates of interoperation. However, it increases the SoS performance if the subsystems are able to interoperate with the new imposed rates with higher performance.

Interoperation $\rightarrow$ Adaptability: An increase in the interoperation might induce an increase in the adaptability if and only if the subsystems are capable to operate with the new imposed rates, otherwise it implies a decrease in that adaptability and the $\mathrm{SoS}$ is not able to return to its predefined level of performance since the new interoperation indicators are not adequate to the new local or external changes. A decrease in the interoperation induces an increase in the SoS adaptability since the restrictions on the subsystems in terms of interoperation indicators are less significant.

The autonomy of the subsystems is the fact to be free to pursue its purpose. That freedom is limited by some constraints. However, those constraints cannot be allowed to overwhelm or violate its capacity or nature to perform. The autonomy is measured based on the classical performance indicators of each subsystem. Autonomy impacts the SoS analysis perspectives as follows:

Autonomy $\rightarrow$ Performance: Each subsystem of the SoS has its own mission(s) to fulfill independently from the overall mission of the SoS. However, if the autonomy of the subsystems increases, its participation in the SoS can be lower which implies a decrease in the SoS performance. Conversely, a decrease in the autonomy of a subsystem makes its participation in the SoS global mission less restrictive.

Autonomy $\rightarrow$ Adaptability: An increase in the subsystems' autonomy imposes more restrictions that prevent the new changes to be absorbed. Therefore, a decrease in the adaptability takes place. However, a decrease in the subsystems' autonomy implies more freedom to react to any changes and to return rapidly to the predefined level of performance, therefore an increase in the adaptability takes place.

Last, the reversibility means that a subsystem may maintain or retrieve easily its autonomy and performance (including positive and/or negative variations that are accepted) at the end of any collaboration. Reversibility has no impact on the analysis perspectives of the SoS, once a subsystem leaves the SoS, it continues its life cycle 
independently from the System of Systems. However, the requirements related to each subsystem have to be verified.

Table 1 shows how the variation in the interoperability requirements level (inducing then measurement of the respect of the requirement by suing one or several of the methods presented before) impacts the analysis perspectives of the SoS.

Table 1 Matrix of the subsystems interoperability's impact on the SoS analysis perspectives (S: Stable, I: Increase, D: Decrease).

\begin{tabular}{ccc}
\hline Interoperability & \multicolumn{2}{c}{ Analysis perspectives } \\
\hline & Performance & Adaptability \\
Compatibility & $\mathrm{S} / \mathrm{D}$ & $\mathrm{S} / \mathrm{D}$ \\
\hline Interoperation & $\mathrm{D} / \mathrm{S} / \mathrm{I}$ & $\mathrm{D} / \mathrm{S} / \mathrm{I}$ \\
& $\mathrm{D} / \mathrm{S} / \mathrm{I}$ & $\mathrm{I}$ \\
\hline & $\mathrm{S} / \mathrm{D}$ & $\mathrm{D}$ \\
Rutonomy & $\mathrm{S} / \mathrm{I}$ & $\mathrm{S} / \mathrm{I}$ \\
\hline
\end{tabular}

\section{Conclusions and perspectives}

This paper illustrated the importance of the interoperability of the subsystems in the control and evolution of the System of Systems. After careful analysis, we realized that there exists a strong linkage between the interoperability and the SoS characteristics and between the interoperability and some other non-functional characteristics of the SoS (analysis perspectives).

The significance of this paper lies in its ability awareness about the need to consider the interoperability prior the assembling of the subsystems. An impact matrix of the interoperability on the analysis perspectives has been proposed, it is a first crucial step towards an effective System of Systems Engineering. It permits the correct control and evolution of the SoS inside uncertain and unknowable environment in which it must operate.

The proposed matrix will serve to allow the evaluation of the impact of the interoperability on the analysis perspectives. This evaluation will be achieved through the simulation which is currently under development.

\section{References}

1. Boardman, J., Sauser, B.: System of Systems - the meaning of of. IEEE/SMC Int. Conf. Syst. Syst. Eng. 118-123 (2006).

2. Jamshidi, M.: System of systems engineering: innovations for the twenty-first century. John Wiley \& Sons (2011).

3. Maier, M.W.: Architecting principles for systems-of-systems. Syst. Eng. 1, 267-284 (1998). 
4. Ackoff, R.L.: Towards a System of Systems Concepts. Manage. Sci. 17, 661-671 (1971).

5. Carney, D., Fisher, D., Place, P.: Topics in Interoperability: System-of-Systems Evolution. Softw. Eng. Institute, Carnegie Mellon Univ. Pittsburgh, PA. (2005).

6. Bilal, M., Daclin, N., Chapurlat, V.: Collaborative Networked Organizations as System of Systems: a model-based engineering approach. IFIP AICT, pro-ve. (2014).

7. Departement of Defense: DoD Architecture Framework Volume I: Definitions and Guidelines. Architecture. I, 1-46 (2007).

8. R.D. Adcock (EIC). Hoboken, N.T.T. of the S.I. of T.: The Guide to the Systems Engineering Body of Knowledge (SEBoK), v. 1.3, , http://www.sebokwiki.org/ [last visited 2014-07-17].

9. International Council on Systems Engineering (INCOSE): INCOSE Systems Engineering Handbook v. 3.2.2. SE Handb. Work. Gr. 1306 (2011).

10.Camarinha-matos, L.M.: Collaborative networks: A mechanism for enterprise agility and resilience. Enterp. Interoperability VI. 1-8 (2014).

11.Ford, T.C., Colombi, J.M., Graham, S.R., Jacques, D.R.: A Survey on Interoperability Measurement. Twelfth Int. Command Control Res. Technol. Symp. (2007).

12.Stevens Intitute Of Technology, Castle Point On Hudson, Hoboken, N. 07030: Report On System Of Systems Engineering. (2006).

13.Pénalva, J.M., Page, E.: SAGACE: La modélisation des systèmes dont la maîtrise est complexe. ILCE. (1990).

14.Grimm, V., Wissel, C.: Babel, or the ecological stability discussions: an inventory and analysis of terminology and a guide for avoiding confusion. Oecologia. 109, 323-334 (1997).

15.Weyns, D., Andersson, J.: On the challenges of self-adaptation in systems of systems. Proc. First Int. Work. Softw. Eng. Syst. - SESoS '13. 47-51 (2013).

16.Bonnefous, Chantal Courtois, A.: Indicateurs de performance. (2001).

17.Interop: Enterprise Interoperability-Framework and knowledge corpus - Final report. INTEROP NoE, FP6 - Contract n 508011, Deliv. DI.3. 1, 1-44 (2004).

18.Kasunic, M.: Measuring Systems Interoperability: Challenges and opportunities. Tech. Note C. 2004 TN 003 Carnegie-Mellon Univ Pittsburgh PA Softw. Eng. INST. (2004).

19.Panetto, H.: Meta-modèles et modèles pour l'intégration et l'interopérabilité des applications d'entreprises de production. Dr. Diss. Univ. Henri Poincaré-Nancy I. (2006).

20.Truptil, S.: Collaborative process design for Mediation Information System Engineering. 6th Int. Conf. Inf. Syst. Cris. Response Manag. (2009).

21.Ruggaber, R.: Athena-Advanced technologies for Interoperability of heterogeneous enterprise networks and their applications. Interoperability Enterp. Softw. Appl. 459-460 (2006).

22.Lavean, G.: Interoperability in Defense Communications. Commun. IEEE Trans. 28, 14451455 (1980).

23.Mensh, D., Kite, R., Darby, P.: A Methodology for Quantifying Interoperability. Nav. Eng. J. 101.3. (1989).

24.Amanowicz, M., Gajewski, P.: Military communications and information systems interoperability. Mil. Commun. Conf. 1996. MILCOM'96, Conf. Proceedings, IEEE. 1, 280-283 (1996).

25.Defense, D. of: C4ISR Architecture Working Group Final Report - Levels of Information System Interoperability (LISI). Washingt. DC OSD(ASD(C3I)) C4ISR AWG. (1998).

26.Leite, M.J.: Interoperability assessment. Proc. 66th Mil. Oper. Res. Soc. Symp. Monterey, CA. (1998).

27.Clark, T., Jones, R.: Organisational interoperability maturity model for C2. Proc. 1999 Command Control Res. Technol. Symp. (1999).

28.Hamilton, J., John, A., Rosen, J.D., Summers, P.A.: An Interoperability Road Map for C4ISR Legacy Systems. Acquis. Rev. Q. 28.Winter. 17-31 (2002). 
29.Andreas, T., Muguira, J.A.: The levels of conceptual interoperability model. Proc. 2003 Fall Simul. Interoperability Work. 7, (2003).

30.Tolk, A.: Beyond technical interoperability-introducing a reference model for measures of merit for coalition interoperability. Old Dom. UNIV Norfolk VA. (2003).

31.Nato: NATO Allied Data Publication 34: NATO C3 Technical Architecture. Nato, Belgium. 2, (2003).

32.Morris, E., Levine, L., Meyers, C., Place, P., Plakosh, D.: System of Systems Interoperability (SOSI): final report (No. CMU/SEI-2004-TR-004). Carnegie-Mellon Univ Pittsburgh PA Softw. Eng. INST. (2004).

33.Stewart, K., Clarke, H., Goillau, P., Verrall, N., Widdowson, M.: Non-technical interoperability in multinational forces. 9th Int. Command Control Res. Technol. Symp. (2004).

34.Kingston, G., Fewell, S., Richer, W.: An organisational interoperability agility model. Def. Sci. Technol. Organ. Canberra. (2005).

35.Ford, T., Colombi, J., Graham, S., Jacques, D.: The Interoperability Score. Proc. Fifth Annu. Conf. Syst. Eng. Res. 1-10 (2007). 